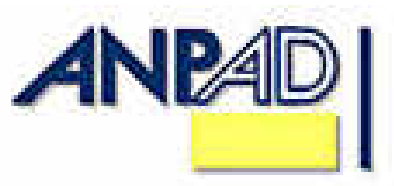

Disponível em http://www.anpad.org.br/rac

RAC, Curitiba, v. 13, n. 1, art. 7, p. 117-135, Jan./Mar. 2009

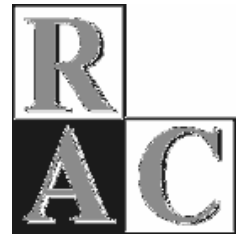

\title{
Similaridade e Dissimilaridade entre Superiores e Subordinados e suas Implicações para a Qualidade da Relação Diádica
}

\section{Similarity and Dissimilarity between Superiors and Subordinates and Their Implications for Dyadic Relationship Quality}

\begin{abstract}
Nereida Salette Paulo da Silveira * Doutoranda em Administração pela Universidade Presbiteriana Mackenzie, São Paulo/SP, Brasil.

Darcy Mitiko Mori Hanashiro Doutora em Administração pela USP. Professora adjunta da Universidade Presbiteriana Mackenzie, São Paulo/SP, Brasil.
\end{abstract}

\footnotetext{
* Endereço: Nereida Salette Paulo da Silveira

Rua da Consolação, 930, Consolação, São Paulo/SP, 01302-907. E-mail: nereidasps@uol.com.br
}

Copyright (C) 2009 RAC. Todos os direitos, inclusive de tradução, são reservados. É permitido citar parte de artigos sem autorização prévia desde que seja identificada a fonte. 


\title{
RESUMO
}

Embora a literatura advogue as vantagens da diversidade da força de trabalho, estudos baseados no Paradigma da Similaridade-Atração indicam uma predisposição das pessoas por sentirem atração por seus semelhantes. Um estudo de campo com dados comparativos de 89 díades buscou investigar os efeitos da similaridade real e percebida na qualidade da relação entre superiores e subordinados sob a ótica da Leader-Member Exchange [LMX]. As características investigadas foram gênero, idade e conflito família-trabalho. Os dados indicam a influência apenas da similaridade percebida na qualidade da relação entre superiores e subordinados. Constatouse também que a satisfação da qualidade e frequiência do contato com o superior (variável moderadora) exerce um efeito de interação com a similaridade percebida na explicação da qualidade da relação diádica. Os procedimentos metodológicos incluíram a análise e validação fatorial de duas escala (EIFT e LMX-7), análise das correlações das variáveis e regressão hierárquica. No final, são discutidas algumas implicações desses resultados e orientações para futuras pesquisas em diversidade.

Palavras-chave: diversidade; percepção de similaridade; relação diádica.

\begin{abstract}
Although literature advocates the advantages of work force diversification, studies based on the SimilarityAttraction Paradigm indicate that people are more disposed to feel attraction to those who are similar to them. A field study with the comparative data of 89 dyads investigated the effect of the actual and perceived similarity in the quality of the relationship between superiors and subordinates within the Leader-Member Exchange [LMX] perspective. The investigated characteristics were: gender, age and work-family conflict. The data indicate the influence only of perceived similarity in the quality of the relationship between superiors and subordinates. This effect broadens when the subordinate feels satisfied with the quality and frequency of contact with his/her superior. The methodological procedures included factorial analysis and validation of two scales (EIFT and LMX-7), the correlations analysis and hierarchic regressions. Finally, the implications of some results and directions for future research in diversity are discussed.
\end{abstract}

Key words: diversity; similarity perception; dyadic relationship. 


\section{INTRODUÇÃO}

Impulsionada por fatores econômicos, aumento da expectativa de vida, alterações nas distribuições de papéis de gênero, aumento da escolaridade das mulheres etc., verifica-se o crescimento da participação de mulheres (Instituto Brasileiro de Geografia e estatística [IBGE], 2008) e pessoas com mais de 50 anos de idade na composição demográfica da força de trabalho nacional ([IBGE], n.d.).

Sob influência de movimentos a favor de melhor qualidade de vida de um lado, e de outro pelo aumento de horas de trabalho efetivamente realizadas (Silva, 2005), também se observam mudanças atitudinais e comportamentais dos trabalhadores em relação à distribuição do tempo e esforços dedicados ao trabalho ou à vida pessoal. Não raro, estas mudanças são acompanhadas de conflitos entre trabalho e família. Estes e outros fatores implicam a ampliação da diversidade no contexto organizacional, seja ela visível (raça, gênero, idade, etc.) ou mais imperceptível (personalidade, valores, balanço trabalho e família, etc.), ou ainda funcional (tempo de empresa, área de atuação, escolaridade, etc.).

A literatura advoga que a diversidade bem administrada favorece as organizações, reduzindo os custos legais e os de rotatividade, melhorando a solução de problemas, ampliando a criatividade e favorecendo a aproximação a novos mercados (Cox \& Blake, 1991; Thomas, 1991, Thomas \& Ely, 1996). Entretanto o ser humano tem maiores dificuldades de lidar com aqueles que lhe parecem diferentes. Percebendo-se como similares, mesmo em condições de desacordo, as pessoas utilizam-se de comportamentos que levam à solução de conflitos, como a negociação e aproximação; mas, contrariamente, quando se percebem como dissimilares, tendem ao afastamento e ao confronto (Triandis, 2003). Os efeitos positivos da similaridade podem também ser compreendidos pelo paradigma da atração-similaridade (Byrne, 1971), para o qual indivíduos similares terão atração interpessoal. Por causa dessa atração os indivíduos experimentarão resultados positivos.

Como decorrência das mudanças na força de trabalho, novas preocupações devem ocupar o cenário organizacional e, dentre estas, notadamente as relações de liderança e seus atores: os superiores e seus subordinados. A teoria de liderança Leader-Member Exchange [LMX] propõe que o relacionamento de superiores e subordinados pode desenvolver-se de forma mais ou menos harmoniosa em conseqüência de diferentes fatores, como, por exemplo, a complementaridade de papéis e a identificação entre ambos. A identificação resulta da percepção de similaridade (ou similaridade real) da díade em certas características.

A percepção da similaridade ou dissimilaridade com o outro pode ser inferida a partir de qualquer atributo (Triandis, 2003), seja este mais visível, como gênero e idade, ou menos visíveis, como o conflito família-trabalho. A maior parte da literatura que investiga o conflito entre família e trabalho propõe que as solicitações de um domínio podem ser incompatíveis com as de outro, gerando conflitos (Davis, Selgrade, Downey, \& Bryant, 2004), que são percebidos em duas direções, do trabalho interferindo na vida familiar e vice-versa (Greenhaus \& Beutell, 1985; Silva, 2005). Ainda que o Conflito Família-Trabalho [CFT] seja de fato vivenciado individualmente, uma similaridade no tipo de conflito experimentado por superiores e subordinados pode criar senso de compartilhamento que leve à percepção de similaridade.

A similaridade e dissimilaridade, contudo, só são importantes na medida em que são percebidas e valorizadas em razão do contexto e do significado para quem percebe (Triandis, 1995, 2003). Assim, não somente as diferenças reais, mas também aquelas percebidas fazem parte da dinâmica da diversidade no contexto organizacional, particularmente na relação entre superior e subordinado.

O presente estudo tem como objetivo analisar a influência da similaridade/dissimilaridade real e percebida nas díades em três atributos: gênero, idade e conflito família-trabalho na qualidade da relação, sob a ótica da LMX. Justifica-se o interesse em entender quais os efeitos da diversidade e sua percepção nestes atributos na qualidade do relacionamento entre superiores e subordinados, pois se espera que os resultados possam trazer luz aos desafios inerentes à diversidade nas organizações e, 
desta forma, contribuir para direcionamento eficaz de políticas de atração e retenção de profissionais, desenvolvimento de práticas que fomentem a melhoria do clima organizacional e direcionamento dos programas de desenvolvimento e capacitação de lideranças.

Supõe-se ainda que a diferenciação de relacionamentos, com base em similaridades em determinados atributos, possa gerar sentimentos de exclusão ou discriminação arbitrária em alguns subordinados, afetando seu desempenho e até de todo o grupo de trabalho. A relação líder-membro de alta qualidade, por sua vez, tem sido associada a melhor desempenho organizacional, aumento do comprometimento de superiores e subordinados, menor rotatividade de profissionais e melhores níveis de avaliação mútua de desempenho (Cortez, 2000).

Um levantamento na produção nacional em 2006 e 2007 apontou a inexistência de estudo que investigasse o efeito das semelhanças e diferenças, percebidas ou reais, no relacionamento entre superiores e seus subordinados. Com o propósito de preencher a lacuna, o artigo busca a produção de conhecimento sobre o papel da diversidade nas relações diádicas verticais de trabalho, a partir de amostra de profissionais de organizações brasileiras.

\section{FUNDAMENTAÇÃO TEÓRICA}

O presente estudo utilizou como base teórica as teorias da Identidade Social e da Autocategorização, o Paradigma da Similaridade-Atração, o conceito de Percepção de Similaridade, a teoria de liderança LMX e a Hipótese do Contato. Essas teorias se complementam para explicar o relacionamento de superiores e subordinados, com base na categorização social e identificação de grupo de pertencimento, a partir da percepção de similaridade ou dissimilaridade.

\section{Percepção de Similaridade}

Percepção é o processo pelo qual indivíduos organizam e interpretam suas impressões sensoriais, a fim de proporcionar sentido ao ambiente que os cerca (Robbins, 2005). O que uma pessoa percebe pode ser substancialmente diferente da realidade objetiva, e o que um indivíduo percebe pode ser bem diferente da percepção do outro sobre o mesmo objeto. Isso se deve ao fato de um conjunto de elementos moldar ou distorcer a percepção.

Dois dogmas estão implícitos na maior parte da pesquisa sobre similaridade: similaridade e diferenças são oposições naturais e a base da similaridade está no compartilhamento de partes ou propriedades dos objetos (Medin, Goldstone, \& Gentner, 1990). Estas pressuposições tratam similaridade como resposta incondicional. A esta perspectiva trataremos como similaridade real. Contudo o que faz com que objetos sejam vistos como parecidos ou diferentes é mais questão relacional e, portanto, implica as referências do sujeito que percebe (Medin et al., 1990). Esta perspectiva denominaremos similaridade percebida. Turban e Jones (1988) definem similaridade percebida como percepções de quão similares são supervisor e subordinado.

Para Tversky (2004) cada objeto é um conjunto de características concretas e abstratas. A avaliação de similaridade pode ser vista como produto da seleção ponderada dessas características comuns entre objetos (Medin et al., 1990). A similaridade dos objetos aumenta na medida em que características comuns são adicionadas ou características distintas são supressas. Entretanto, quando observadas em relação a um contexto específico, apenas algumas das características são relevantes. Portanto, elementos relacionados seja ao objeto seja ao alvo da percepção, ao observador como ao contexto condicionam quais características são salientes e relevantes para a avaliação de similaridade e de dissimilaridade e o peso relativo dessas características (Tversky, 2004).

A percepção de similaridade é um dos processos contribuintes para a percepção social, Por consequiência, a percepção de pessoas é também influenciada por processos internos e externos ao 
indivíduo, o que leva a inferências sobre comportamentos, motivações, atitudes e atribuição de intencionalidades. Para Zebrowitz (1990) as percepções das pessoas afetam não apenas o comportamento em relação a elas, mas também contribuem para o comportamento delas próprias, como, por exemplo, o efeito de profecia auto-realizada (Aronson, Wilson, \& Akert, 2002). Portanto a percepção de similaridade ou dissimilaridade é determinante no estabelecimento e desenvolvimento das relações interpessoais (Triandis, 2003).

A distinção entre similaridade-dissimilaridade real ou percebida se faz importante na medida em que a pesquisa sobre a influência da similaridade em relações diádicas de superiores e subordinados mostra que os efeitos da similaridade percebida são mais fortes que o da similaridade real (Hobman, Bordia, \& Gallois, 2004; Strauss, Barrick, \& Connerley, 2001). Uma das razões que as percepções de similaridade predizem mais consistentemente resultados relacionados ao trabalho do que a similaridade real é que as "pessoas reagem com base na percepção da realidade e não na realidade per si" (Strauss et al., 2001). Em pesquisa de campo, realizada por Strauss (1993), a similaridade real de personalidade (entre avaliador e avaliado) não estava relacionada significativamente com o desempenho. Entretanto a autora encontrou forte suporte para a relação entre a percepção de similaridade (personalidade e global) e desempenho. Esses resultados sugerem que o efeito similar a mim de Byrne (1971) existe no contexto de avaliação de desempenho para a percepção de similaridade e não para a similaridade real. Quando determinada dimensão é mais ou menos valorizada em determinados contextos, maior será a probabilidade da percepção de similaridade ou dissimilaridade nesta dimensão ser percebida (Triandis, 1995), fundamentada na teoria da autocategorização (Turner, 1987). Além do mais, a similaridade não é fenômeno simétrico (Tversky, 2004) e, como seu direcionamento ocorre em função da saliência do objeto, é admissível que a percepção de similaridade do liderado em relação ao líder seja maior que o inverso.

\section{Paradigma da Similaridade-atração}

Estudos indicam que, em sendo constantes as demais variáveis, por meio da percepção da similaridade em atitudes (Wexley, Alexander, Greenawalt, \& Couch, 1980), características demográficas (Tsui, Egan, \& O'Reilly, 1992), personalidade (Saltz, 2004; Strauss et al., 2001), probabilidade de relacionamento (Aron, Steele, Kashdan, \& Perez, 2006), dentre outras, desenvolve-se a atração entre pessoas. A este fenômeno da atração pelo semelhante, Byrne (1971) denominou Paradigma da Similaridade-Atração.

As atitudes, quando declaradas, eliciam respostas afetivas positivas ou negativas. A similaridade de atitudes proporciona maior previsibilidade de comportamento, comunicação mais fluida, e maior grau de concordância com o outro. Essas condições propiciam interações mais recompensadoras; a atração entre as pessoas ocorre com maior naturalidade, quando as interações são recompensadoras (Byrne, 1971).

Byrne não distingue entre similaridade real, que se refere à correspondência de atributos entre dois objetos, e a percebida (Garcia, 2004). Para o autor similaridade é vista tanto como uma correspondência objetiva entre duas pessoas, como o reconhecimento da similaridade. Esse reconhecimento traduz um raciocínio subjacente de que a similaridade é fenômeno constituído por aspectos reais e percebidos. Similaridade ou dissimilaridade real diz respeito ao compartilhamento de características concretas e objetivas entre indivíduos, sejam elas prontamente visíveis ou apenas passíveis de identificação após um período de interação, como, por exemplo, atitudes. Embora a similaridade real e a percebida estejam relacionadas (Harrison, Price, \& Bell, 1998; Strauss et al., 2001), fatores pessoais e contextuais alteram a percepção, fazendo com que determinadas características possam ser mais ou menos salientes para alguns indivíduos, sugerindo que a similaridade real pode não ser igual à similaridade percebida.

$\mathrm{Na}$ ausência de maiores informações sobre as atitudes, a forma de pensar e agir dos outros, as pessoas tendem a fundamentar seus julgamentos nas características manifestas do outro, como gênero, raça, aparência, baseando-se em simbolismos e experiências prévios associados a essas características, 
representando de forma prototípica grupos de pessoas que reúnem características comuns, como pertencentes a uma determinada categoria social.

\section{Teorias da Identidade Social e Autocategorização}

O processo de categorização social é explicado pelas Teorias da Identidade Social [TIS] e da Autocategorização [TAC], desenvolvidas respectivamente por Henry Tajfel e John Turner, que trouxeram contribuições complementares à abordagem da identidade social. De acordo com a TAC, a partir da percepção de compartilhamento de determinadas características, as pessoas categorizam a si mesmas e aos demais (Ashfort \& Mael, 1989). Segundo a TIS, com base nessas categorizações e da identificação ou não com tais traços, os indivíduos desenvolvem identificações de pertencimento (endogrupo) e não pertencimento (exogrupo) a determinados grupos sociais. A identidade social proporciona ao indivíduo, juntamente com a identidade pessoal, a autodefinição que deriva desse reconhecimento de ser parte de um ou vários grupos sociais, juntamente com o significado emocional e de valor atribuído a esta filiação.

A extensão em que o indivíduo se define no nível pessoal ou social é ajustável em relação ao ambiente. Desse modo, uma pessoa não tem uma, mas, sim, várias identidades sociais que correspondem aos diversos grupos a que pertence, e que são ativadas de acordo com as condições contextuais. A percepção de similaridade entre duas pessoas depende não apenas de que essas pessoas compartilhem determinadas características, mas também da relevância e saliência dessas características no contexto específico e o reflexo dessas características em sua própria representação (Ferdman, 1995).

A identificação com os grupos de pertencimento é base para o desenvolvimento de identidade social positiva, em razão da avaliação positiva projetada no grupo de pertencimento e da identificação negativa projetada no exogrupo (Machado, 2003). A identificação social reforça a percepção de fronteiras de grupo, propiciando o sentimento de nós contra eles (Hogg \& Terry, 2000). No contexto organizacional grupos podem ser formados em razão da estrutura formal de distribuição de tarefas, produtos, processos ou localização geográfica, tornando o ambiente organizacional a perfeita arena para explicar o comportamento de grupo e como este processo influencia as relações (Hogg \& Terry, 2001).

\section{Relacionamento Líder-Membro - LMX}

A LMX, inicialmente denominada Ligação Diádica Vertical (Vertical Dyad Linkage [VDL]), é uma teoria de liderança baseada no conceito de que a relação interpessoal de superior e subordinado se dá de forma única com cada subordinado. Embora a relação se dê no nível diádico, ela parte da categorização mútua de superiores e subordinados em relação a grupos de identidade social. Superiores categorizam seus subordinados em contínuo pertencimento a seu grupo, tomando por base similaridades em determinados atributos, visíveis ou não, reais ou apenas percebidos. O relacionamento a partir daí se desenvolve por meio de trocas ou permutas diferenciadas com o endogrupo e exogrupo. Na suposição de que recursos dos superiores, tais como remuneração, tempo ou informações são escassos, a distribuição desses recursos se dá de forma desigual entre os subordinados (Yukl, 2001). Considerando que a maioria das organizações possui hierarquia piramidal que impõe, mesmo ao líder mais autocrático, a necessidade de delegação (Yu \& Liang, 2004), aos subordinados pertencentes ao endogrupo são fornecidos mais recursos, atenção e oportunidade de desenvolverem tarefas mais atraentes. Em movimento recíproco, esses subordinados retribuem com melhor desempenho e lealdade. Contrariamente, os subordinados do exogrupo são contemplados apenas com as condições mínimas de direito e retribuem estritamente com as atribuições formais previstas em seus cargos (Graen \& Uhl-Bien, 1998; Liden \& Graen, 1980). À qualidade dessa interação da díade atribui-se o termo qualidade da LMX, expressão doravante usada no artigo. Com fundamentação no paradigma da Similaridade-Atração (Byrne, 1971) e nas teorias da Identidade Social e Autocategorização, sugere-se a relação entre similaridade e qualidade da LMX, dando origem à primeira hipótese da pesquisa de campo. 
Hipótese 1: A similaridade real (gênero, idade e conflito família-trabalho) e a de similaridade percebida estão positivamente correlacionadas à qualidade da relação de superiores e subordinados, sob a ótica da LMX.

Uma distinção encontrada na literatura diz respeito à percepção ou realidade das características que subsidiam o processo de categorização de superiores e subordinados (Garcia, 2004; Strauss et al., 2001; Wexley et al., 1980). Nem todas as características são percebidas com a mesma intensidade. A saliência de determinadas características em relação a outras está relacionada à valorização desta em função do contexto (Haslam \& Platow, 2001).

Para Miller (2002), o relacionamento entre membros de grupos distintos de pertencimento pode evoluir para o relacionamento interpessoal, por meio da obtenção e comparação de informações que levam ao descobrimento de características mútuas valorizadas no contexto específico. Tal processo pressupõe abertura e fornecimento voluntário de informações pessoais, por conta da confiança depositada no outro, ampliando a percepção de similaridade. A percepção de similaridade, por sua vez, base para a identificação de membros de endogrupo e exogrupo, determina a qualidade do relacionamento pela ótica da LMX, ou seja, este processo resulta em mudanças na categorização dos membros de grupo de pertencimento porque, embora a categoria social inicial ainda exista, os traços salientes e a percepção de similaridade são modificados. Desta forma propõe-se a segunda hipótese do estudo.

Hipótese 2: A similaridade percebida exerce maior influência na Qualidade da LMX que a similaridade real.

\section{Hipótese do Contato}

Allport, em sua obra The Nature of Prejudice de 1954 formulou a Hipótese do Contato, defendendo que o contato repetido com membros do exogrupo pode ter efeito positivo nos estereótipos e preconceitos. Porém Allport não fala de mero contato, e sim que por meio de certas condições que levam ao descobrimento mútuo de similaridades essenciais, reduzem-se os comportamentos hostis originados pelo preconceito e estereótipo. As condições primeiramente descritas por Allport e desde então submetidas à verificação empírica são: (a) igual status dos grupos no contexto de interação; (b) metas comuns; (c) cooperação entre os grupos; e (d) apoio de autoridades e instituições (Pettigrew \& Tropp, 2006). O contato é recompensador, portanto satisfatório, se a percepção de dissimilaridade for substituída pela percepção de similaridade, pela verificação do pertencimento dos envolvidos em uma categoria social comum (Lynch, 2001; Triandis, 1995).

Dois aspectos influenciam a positividade da interação: a frequiência (oportunidade de interações) e a qualidade do contato (voluntariedade, cooperação, igualdade ou desigualdade de importância dos envolvidos etc.) (Triandis, 1995).

Para Triandis (2004) o contato resulta em emoções e atitudes positivas entre as partes, quando há percepção de similaridade; contudo as conseqüências são contrárias, quando há percepção de dissimilaridade; portanto a frequiência e a qualidade do contato podem modificar a relação entre as características de diversidade (similaridade e dissimilaridade), real ou percebida e a qualidade da relação. O contato pode desempenhar o papel de variável moderadora, gerando a terceira hipótese.

Hipótese 3: O contato satisfatório entre a díade supervisor-subordinado, em termos de frequiência e qualidade, altera positivamente a relação entre Similaridade percebida e a Qualidade da LMX.

As variáveis relacionadas acima constituem uma estrutura conceitual representada na Figura 1. 


\section{Figura 1: Modelo Relacional Proposto - Similaridade Real e Percebida e Qualidade da LMX}

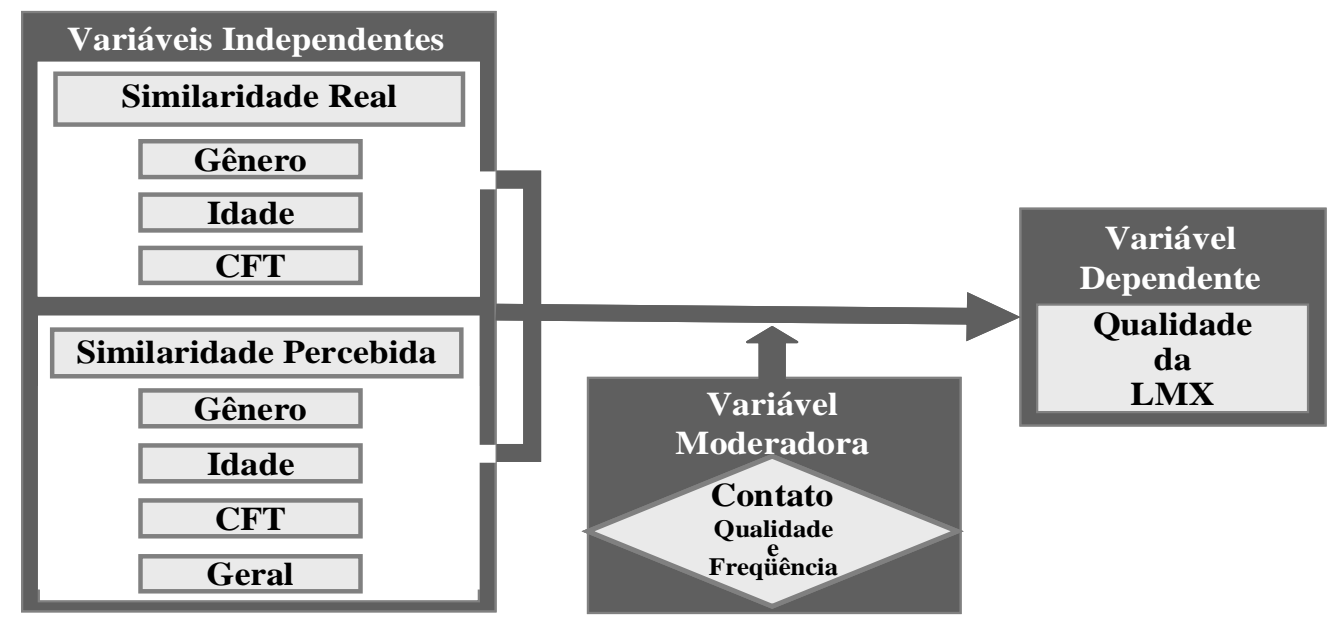

\section{PROCEDIMENTOS METOdOLÓGICOS}

\section{Amostra}

O nível de análise diádico encerra algumas dificuldades no planejamento amostral. De fato, torna-se impraticável a aplicação de uma amostra aleatória, pela necessidade de constituir díades formadas pelo superior e seu respectivo subordinado. Ambos precisam estar comprometidos em responder. Quando um deles falha, perde-se a díade. Por esse motivo, aliado à falta de acesso de se realizar a pesquisa em uma única organização (onde talvez pudesse tentar uma amostra aleatória), este estudo optou pela adoção de uma amostra por acessibilidade, ciente de todas as limitações que lhe são peculiares, no que tange à generalização dos dados. Logo, os resultados apresentados expressam a realidade das díades estudadas, mas não tem a intenção de expandir suas conclusões para qualquer díade funcional.

As díades foram compostas por profissionais de distintos níveis hierárquicos e atuantes em empresas pertencentes a diferentes setores. Pela necessidade de conhecimento mínimo sobre aspectos além daqueles estritamente profissionais, considerou-se na amostra a relação formal de período superior a de um ano.

\section{Procedimentos}

Questionários impressos foram distribuídos pessoalmente aos respondentes, sendo uma versão para o superior e outra para os subordinados, em envelopes separados, selados e endereçados para retorno pelo correio. A necessidade de caracterização diádica foi sempre ressaltada, de forma que se solicitava ao respondente entregar o questionário complementar ao seu superior ou subordinado. Foram distribuídos 320 questionários, dos quais 140 para superiores e 180 para subordinados. Desses, retornaram 137, distribuídos em 48 superiores e 89 subordinados. Essa diferença se deve ao fato de vários superiores terem encaminhado o questionário para a equipe inteira. Para comparação diádica, as respostas desses superiores foram repetidas pelo número de subordinados, compondo uma amostra de 89 díades. Esse número pode expressar uma amostra pequena. No entanto os estudos realizados em díades para avaliação da qualidade da LMX não exibem amostras muito amplas. Por exemplo, Greene (1972) utilizou 141 díades; Liden e Graen (1980) 42; Duarte, Goodson e Klich, (1994) 261; Bauer e Green (1996) 205; Engle e Lord (1997) 76; Phillips e Bedeian (1994) 84; Deluga (1998) 127; Cortez (2000) 135; Huang e Iun (2006) 205. 


\section{Medidas}

As variáveis independentes do modelo foram constituídas, originalmente, por gênero, idade e conflito família/trabalho. Para a análise estatística, essas variáveis sofreram transformações, visando à obtenção do valor de similaridade/dissimilaridade na dimensão real de cada uma das 89 díades da amostram, como também na dimensão percebida na ótica dos subordinados.

\section{Gênero 'Real'}

A similaridade, ou seja, díades do mesmo gênero (H/H e M/M) foram codificadas com o valor zero (0) e a dissimilaridade, díades de diferentes gêneros, com o valor um (1). Tal comparação resultou em 55 díades similares e 34 dissimilares.

\section{Idade 'Real'}

Pela comparação direta entre as idades, dificilmente obteríamos similaridade das díades. Markert (2004) defende que a divisão de faixas etárias em intervalos de 10 anos é aceita como exercendo uma função transformadora do ponto de vista psicobiológico nas pessoas. Seguindo essa ótica, foram criadas faixas etárias de 10 em 10 anos, a partir de 20 anos de idade. A similaridade, ou seja, díades pertencentes à mesma faixa etária foram codificadas com o valor $(0)$ e a dissimilaridade, díades pertencentes a diferentes faixas etárias, foram codificadas com o valor (1). Tal comparação resultou em 26 díades similares e 63 dissimilares.

\section{Conflito Família-trabalho [CFT] 'Real'}

Define-se CFT como a tensão resultante do investimento de tempo destinado ao trabalho ou à família, da incompatibilidade gerada pelo desempenho de papéis relativos a um ou outro domínio e do antagonismo entre compromissos exigidos entre a vida profissional e familiar.

O CFT foi medido pela Escala de Interação Trabalho-Família, criada e validada por Paschoal, Tamayo e Bahram (Paschoal \& Tamayo, 2005). Compõe-se de 14 variáveis subdivididas em dois fatores: impacto da família sobre o trabalho e o impacto do trabalho sobre a família, conforme a teoria do transbordamento (Greenhaus \& Beutel, 1985; Silva, 2005). Cada fator é formado por sete itens ancorados em uma escala de frequiência, variando de um (nunca) a cinco (quase sempre). Esses dois fatores não foram validados na amostra de 137 sujeitos (total de respondentes, visto não haver necessidade de considerar os dados diádicos).

Observados todos os procedimentos de fatorabilidade e atendidos seus requisitos fundamentais (Hair, Anderson, Tatham, \& Black, 2006), após várias tentativas de análise fatorial com diferentes métodos de rotação, a melhor solução, ou seja, com fatores interpretáveis e menor número de itens representando complexidade (participando simultaneamente de dois ou três fatores) foi obtida por rotação oblíqua, extração por componentes principais, com definição prévia de dois fatores. O fator um apresentou 31,4\% de variância explicada e expressa um tipo de resposta ao CFT que atua sobre aspectos emocionais e de ânimo, tirando do indivíduo sua concentração, atenção e bom humor. $\mathrm{O}$ fator dois, com 12,6\% de variância explicada, reflete o conflito resultante da impossibilidade de estar presente em dois locais e situações ao mesmo tempo, exigindo do indivíduo que opte por sacrificar uma das esferas de sua vida, profissional ou familiar. As 14 variáveis foram preservadas na nova estrutura fatorial. 
A consistência interna, medida pelo alfa de Cronbach, do fator 1 e 2 foi 0,770 . Os itens de cada fator foram somados, para superiores e subordinados. A diferença entre esses valores das díades foi considerada para a análise estatística como o grau de dissimilaridade, na medida em que a comparação das díades tende muito mais a resultados diferentes que semelhantes.

\section{As Variáveis de Percepção}

Além de tratar da similaridade e dissimilaridade real de Gênero, Idade e CFT, comparando os valores das díades, o modelo da pesquisa inclui também a dimensão percebida. Desse modo, esses mesmos atributos foram mensurados por meio de quatro itens em que o subordinado avaliava, genericamente, o grau de similaridade sua em relação ao jeito de pensar de seu superior quanto ao Gênero, Idade, CFT e uma percepção de similaridade geral. Os itens foram mensurados por meio de uma escala que variava de cinco (5), muito parecido; até um (1), muito diferente. Não foi necessário transformação nessa variável, pois os itens foram respondidos somente pelos subordinados.

\section{Variável Dependente: Qualidade da Relação}

Foi mensurada pela escala LMX-7, desenvolvida em 1982 por Graen, Novak e Summerkamp (como citado por Graen \& Uhl-Bien, 1998) e, segundo os autores, a mais usada para medir a qualidade da relação superior-subordinado. A escala foi traduzida seguindo os preceitos recomendados pela técnica de back translation (Brislin, 1970). A escala foi respondida somente pelos subordinados (89 sujeitos). Visando à validação na amostra pesquisada, foi realizada a análise fatorial, rotação oblíqua, extração de fatores por meio de componentes principais com eingenvalue $>1$. O teste de esfericidade foi significativo, porém, o KMO foi ruim $(0,594)$, conforme Hair et al. (2006). A análise da matriz de anti-imagem indicou a eliminação de dois itens. Retiradas essas variáveis da escala, nova fatorial foi realizada, gerando apenas um fator, com $66,7 \%$ de variância total explicada e alpha de Cronbach de 0,858 . Visando melhorar a consistência interna, decidiu-se pela eliminação de mais um item. Nova fatorial foi realizada, confirmando um único fator, formado por quatro itens, com variância explicada de $79,1 \%$ e alfa de Cronbach de 0,909. A partir desse resultado, utilizou-se a escala somada para efeito das análises estatísticas.

\section{Variável Moderadora}

O contato foi identificado como variável moderadora, fundamentado na Hipótese de Contato (Pettigrew, 1998). Essa variável foi medida por duas assertivas criadas especificamente, com valores dicotômicos: (2) satisfeito e (1) insatisfeito com a frequiência e a qualidade do contato.

\section{Pré-teste dos Instrumentos}

Todos os instrumentos criados ou desenvolvidos por outros autores foram objetos de análise semântica e pré-testes com alunos e professores de mestrado em Administração de Empresas e integrantes do Grupo de Diversidade.

\section{RESULTADOS}

\section{Caracterização da Amostra Pesquisada}

Os homens constituíram $60 \%$ da mostra, entre os quais $56 \%$ figuraram como superiores e $62 \%$ como subordinados, enquanto dos $40 \%$ de mulheres, $44 \%$ exerciam cargo de comando de pessoas, ou seja, superiores, e 38\% subordinadas. Quanto à distribuição de idade, os superiores de ambos os gêneros 
concentraram-se nas faixas de 30 a 39 (38\%) e de 40 a 49 (48\%) anos, totalizando 86\%, enquanto os subordinados apresentaram-se distribuídos quase uniformemente entre as faixas que vão de 20 a 29 (35\%), 30 a $39(33 \%)$ e 40 a 49 (26\%) anos, totalizando $97 \%$ dos respondentes. Nas díades dissimilares em relação a gênero, ocupando a posição de comando, encontraram-se 10 mulheres $(35,8 \%)$ e 18 homens $(34,2 \%)$. Nas díades similares, a distribuição ficou em 16 mulheres $(26,2 \%)$ e 45 homens $(73,8 \%)$ em cargo superior.

\section{Matriz de Intercorrelação}

Buscando compreender como e em que intensidade as variáveis do modelo se relacionavam entre si, foi realizada uma correlação (Pearson).

Tabela 1: Matriz de Intercorrelações

\begin{tabular}{|c|c|c|c|c|c|c|c|c|c|c|c|}
\hline & VI-1 & VI-2 & VI-3 & VI-4 & VI-5 & \multicolumn{2}{|c|}{ VI-6 } & VI-7 & VI-8 & VM-9 & VM-10 \\
\hline \multicolumn{12}{|l|}{ VI-1 } \\
\hline VI-2 & 010 & & & & & & & & & & \\
\hline VI-3 &, 150 &,- 024 & & & & & & & & & \\
\hline VI-4 & ,017 & ,121 &, $320 * *$ & & & & & & & & \\
\hline VI-5 & ,099 & ,117 & , 130 &, 150 & & & & & & & \\
\hline VI-6 & , 135 & , 157 &, 154 &, 111 &, $737 * *$ & & & & & & \\
\hline VI-7 &,- 005 & ,081 & , 109 & ,040 &, $443 * *$ &, 482 & & & & & \\
\hline VI-8 & , 120 &, 144 & , 188 & ,046 &, $683 * *$ &, 588 & &, $556 * *$ & & & \\
\hline VM-9 & ,062 & , 196 & ,119 &, 183 &, $295 * *$ & ,25 & & ,172 & ,261* & & \\
\hline VM10 &,- 147 &,- 011 & 177 &, 155 & ,266* & ,21 & & ,226* & 129 &, $639 * *$ & \\
\hline VD-LMX & ,019 &,- 002 & ,256* & ,105 &, $393 * *$ & ,511 & &, $309 * *$ &, $364 * *$ &, $481 * *$ &, $404 * *$ \\
\hline \multicolumn{12}{|c|}{$\begin{array}{l}\text { ** Correlação (Pearson): significante ao nível } 0.01 \\
\text { * Correlação (Pearson): significante ao nível } 0.05 \\
\text { Legenda }\end{array}$} \\
\hline $\begin{array}{ll}\text { VI-1 } & \text { Simila } \\
\text { VI-2 } & \text { Simila } \\
\text { VI-3 } & \text { Simila } \\
\text { VI-4 } & \text { Simila } \\
\text { VI-5 } & \text { Simila } \\
\text { VI-6 } & \text { Simila }\end{array}$ & $\begin{array}{l}\text { dade rea } \\
\text { dade rea } \\
\text { dade rea } \\
\text { dade rea } \\
\text { dade per } \\
\text { dade per }\end{array}$ & $\begin{array}{l}\text { de gêne } \\
\text { de idade } \\
\text { de CFT } \\
\text { de CFT } \\
\text { ebida de } \\
\text { ebida de }\end{array}$ & $\begin{array}{l}\text { Fator1 } \\
\text { Fator2 } \\
\text { ênero } \\
\text { dade }\end{array}$ & & $\begin{array}{l}\text { VI-7 } \\
\text { VI-8 } \\
\text { VM } \\
\text { VM } \\
\text { VD- }\end{array}$ & & $\begin{array}{l}\text { Sin } \\
\text { Sin } \\
\text { Sat } \\
\text { Sat } \\
\text { Ou }\end{array}$ & $\begin{array}{l}\text { laridade } p \\
\text { laridade } p \\
\text { facão com } \\
\text { facão com } \\
\text { idade da I }\end{array}$ & $\begin{array}{l}\text { ebida de } \\
\text { ebida ge } \\
\text { ualidade } \\
\text { requiênci } \\
\text { X }\end{array}$ & $\begin{array}{l}\text { FT } \\
\text { o Contato } \\
\text { lo Contato }\end{array}$ & \\
\hline
\end{tabular}

Observa-se que a dimensão real não apresentou correlação significativa, exceto com o CFT, fator 2. No entanto a dimensão percebida correlacionou-se entre si, com a qualidade do contato (moderadora) e com a Qualidade da LMX (dependente), conforme pode ser observado na Tabela 1.

A seguir serão apresentados os resultados de cada hipótese e, subseqüentemente, elas serão discutidas à luz das teorias que as fundamentaram.

Hipótese 1. Foi realizada regressão linear múltipla com todas as variáveis reais e percebidas em relação à Qualidade da LMX. Usando o método Enter, emergiu um modelo significante $\left(\mathrm{F}_{8,80}=4,492\right.$, $\mathrm{p}<0,001), \mathrm{R}^{2}=0,310$, ou seja, a medida da proporção da variância da Qualidade da LMX (VD), que é explicada pelas variáveis independentes (VI) ou preditoras. Esse valor reflete que, no conjunto, as VI explicam pouco as variações da VD. Analisando-se os coeficientes Betas, apenas a variável similaridade percebida de idade foi significativa para $\mathrm{p}<0,002, \operatorname{com} \beta$ padronizado $=0,455$.

Hipótese 2. Foram comparadas, par a par, todas as variáveis referentes à similaridade ou dissimilaridade em características reais com a variável de percepção correspondente, até mesmo os dois fatores de conflito família-trabalho. Também foi comparada a variável Percepção de Similaridade Geral com todas as variáveis de similaridade real e, finalmente, foram comparadas em conjunto todas as variáveis referentes à similaridade/dissimilaridade real com todas as de percepção. Desta forma, foram realizadas sete regressões hierárquicas, com o ingresso primeiramente da variável de percepção, 
pois já demonstrou ter maior poder explicativo na variação da Qualidade da LMX, pela observação da matriz de intercorrelação, e posterior ingresso da variável de característica real.

No modelo 1, verificou-se que, para as sete regressões, as estatísticas F com 8 e 77 graus de liberdade foram significativas para $\mathrm{p}<0,01$, conforme se observa na Tabela 2. A introdução da dimensão real (modelo 2) das respectivas variáveis resultou em estatísticas $\mathrm{F}_{8,77}$ (change) não significativa para $\mathrm{p}<0,01$, embora $\mathrm{o} \mathrm{R}^{2}$ não tenha reduzido. Isso significa que as variáveis de percepção têm poder explicativo superior àquelas reais, como preditoras da Qualidade da LMX.

Tabela 2: Modelo de Regressão Hierárquica - Percepção de Similaridade e S/D Real na Qualidade da LMX

\begin{tabular}{lccc}
\hline Modelo 1 & & & \\
\hline \multicolumn{1}{l}{ Percepção de Similaridade } & & & \\
\hline Gênero & $\mathbf{R}^{2}$ & $\mathbf{F}$ & Sig F \\
\hline Idade & 0,155 & 13,923 & 0,000 \\
\hline CFT & 0,261 & 30,704 & 0,000 \\
\hline CFT & 0,095 & 9,179 & 0,003 \\
\hline CFT & 0,095 & 9,179 & 0,003 \\
\hline Geral & 0,095 & 9,179 & 0,003 \\
\hline Percepção (todas) & 0,133 & 13,298 & 0,000 \\
\hline & 0,269 & 7,734 & 0,000 \\
\hline
\end{tabular}

\begin{tabular}{lcccc}
\hline \multicolumn{4}{l}{ Modelo 2 } \\
\multicolumn{4}{l}{ Percepção de Similaridade e Similaridade/Dissimilaridade Real } \\
\hline & $\mathbf{R}^{2}$ & $\begin{array}{l}\text { Sig F } \\
\text { (change) }\end{array}$ & F & Sig F \\
\hline Gênero & 0,155 & 0,842 & 7,893 & 0,001 \\
\hline Idade & 0,268 & 0,370 & 15,726 & 0,000 \\
\hline CFT Fator 1 & 0,145 & 0,027 & 7,318 & 0,001 \\
\hline CFT Fator 2 & 0,104 & 0,365 & 4,994 & 0,009 \\
\hline CFT Fatores 1 e 2 & 0,146 & 0,087 & 4,843 & 0,004 \\
\hline Gênero, Idade e CFT & 0,175 & 0,380 & 3,518 & 0,006 \\
\hline Real (todas) & 0,310 & 0,325 & 4,492 & 0,000 \\
\hline
\end{tabular}

Hipótese 3. Observou-se que (modelo 1) para as quatro regressões hierárquicas, as estatísticas $\mathrm{F}$ com 8 e 77 graus de liberdade foram significativas para $p<0,01$ (Tabela 3). A introdução da variável Qualidade do contato no modelo 2 resultou em estatísticas $\mathrm{F}_{8,77}$ (change) significativa para $\mathrm{p}<0,01$. Conclui-se que a Qualidade do contato exerce de fato um papel moderador, influenciando a relação entre as variáveis independentes de percepção e a Qualidade da LMX.

Tabela 3: Modelo de Regressão Hierárquica - Efeito Moderador da Qualidade do Contato na Percepção de Similaridade

Modelo 1- Similaridade Percebida

\begin{tabular}{lccc}
\hline \hline & $\mathbf{R}^{\mathbf{2}}$ & $\mathbf{F}$ & Sig $\mathbf{~}$ \\
\hline Gênero & 0,155 & 15,923 & 0,000 \\
\hline Idade & 0,261 & 30,704 & 0,000 \\
\hline CFT & 0,095 & 9,179 & 0,003 \\
\hline Geral & 0,133 & 13,298 & 0,000 \\
\hline \hline
\end{tabular}

Modelo 2 - Similaridade Percebida Moderada

\begin{tabular}{rrrrr}
\hline \hline & \multicolumn{5}{c}{ Sig } \\
$\mathbf{R}^{\mathbf{2}}$ & $\mathbf{F}($ change) & $\mathbf{F}($ change) & $\mathbf{F}$ & Sig F \\
\hline 0,298 & 17,588 & 0,000 & 18,237 & 0,000 \\
\hline 0,400 & 19,910 & 0,000 & 28,644 & 0,000 \\
\hline 0,282 & 22,292 & 0,000 & 16,859 & 0,000 \\
\hline 0,292 & 19,338 & 0,000 & 17,719 & 0,000 \\
\hline \hline
\end{tabular}


Tabela 4: Modelo de Regressão Hierárquica - Efeito Moderador da Frequiência do Contato na Percepção de Similaridade

\begin{tabular}{lccc}
\multicolumn{4}{c}{ Modelo 1 - Similaridade Percebida } \\
\hline \hline & $\mathbf{R}^{\mathbf{2}}$ & $\mathbf{F}$ & Sig F \\
\hline Gênero & 0,155 & 15,923 & 0,000 \\
\hline Idade & 0,261 & 30,704 & 0,000 \\
\hline CFT & 0,095 & 9,179 & 0,003 \\
\hline Geral & 0,133 & 13,298 & 0,000 \\
\hline \hline
\end{tabular}

Modelo 2 - Similaridade Percebida Moderada

\begin{tabular}{crccc}
\hline \hline & \multicolumn{5}{c}{ Sig } \\
$\mathbf{R}^{\mathbf{2}}$ & F(change) & $\mathbf{F}$ (change) & $\mathbf{F}$ & Sig $\mathbf{~}$ \\
\hline 0,241 & 9,809 & 0,002 & 13,672 & 0,000 \\
\hline 0,339 & 10,154 & 0,002 & 20,044 & 0,000 \\
\hline 0,215 & 13,125 & 0,000 & 11,807 & 0,000 \\
\hline 0,275 & 16,824 & 0,000 & 16,270 & 0,000 \\
\hline \hline
\end{tabular}

Os resultados similares foram obtidos para a variável Freqüência do contato, observados na Tabela 4. Verifica-se, no entanto, que a Qualidade do contato apresentou poder explicativo superior em relação à Qualidade da LMX.

O resultado dos testes das hipóteses da pesquisa conduziu a algumas mudanças no modelo proposto, representado graficamente conforme Figura 2.

\section{Figura 2: Modelo Relacional Verificado - Similaridade ‘Real' e 'Percebida' e Qualidade da LMX}

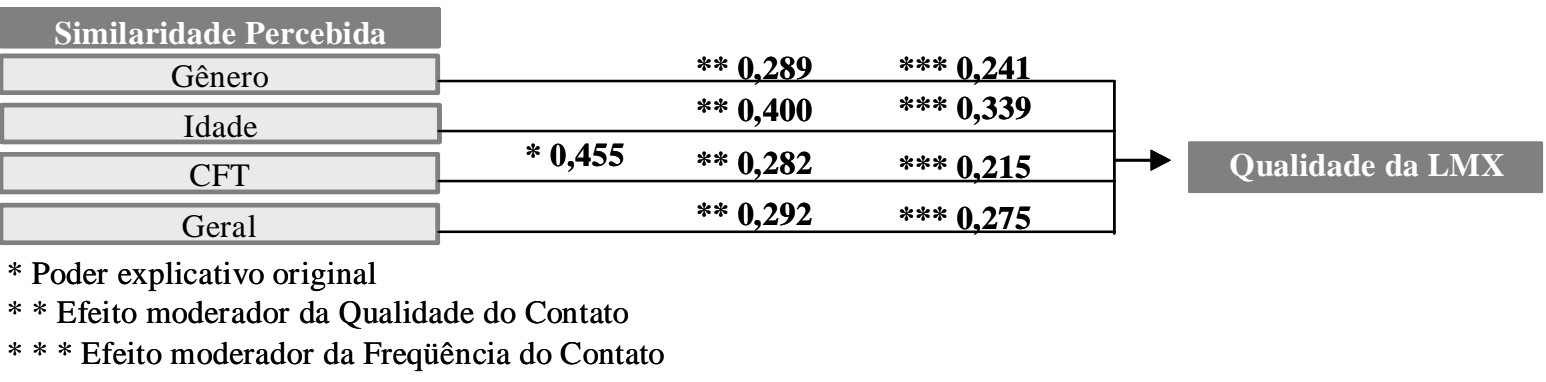

\section{Discussão dos Resultados e Conclusão}

Por um lado, a similaridade tem papel importante na formação de categorias (Sloutsky, 2003) e a categorização é processo necessário à limitada capacidade humana de lidar com variedade muito grande de informações (Triandis, 2003); por outro lado, a percepção de similaridade desempenha função mais efetiva, para que o relacionamento diádico evolua da relação intergrupal para a interpessoal. De modo geral, a percepção de similaridade facilita a interação, pois sugere semelhança nos padrões de pensamento e de pressupostos básicos (Triandis, 2003), dando condições para o desenvolvimento de maior reciprocidade e essa, por sua vez, é subjacente à qualidade da relação entre superiores e subordinados, sob a ótica da LMX. Entretanto o que de fato leva à percepção de similaridade ou dissimilaridade é ainda um terreno merecedor de exploração (Medin et al., 1990). Existem evidências de que a similaridade real é um subsídio para a percepção de similaridade, mas não há relação direta entre ambas (Pulakos \& Wexley, 1983), como se observa na matriz de correlação. É, contudo, consensual que o contexto exerce papel fundamental para a saliência de determinadas características, o que facilita sua percepção (Haslam \& Platow, 2001; Hogg \& Terry, 2000; Triandis, 1995).

$\mathrm{O}$ presente estudo procurou investigar em que medida a similaridade ou dissimilaridade real e percebida influenciam a qualidade da relação entre superiores e subordinados, partindo dos pressupostos do Paradigma da Similaridade-Atração, que especifica que a similaridade entre as 
pessoas leva à atração, na medida em que é reforçadora. Os achados apontam a influência do grau de similaridade/dissimilaridade percebida na qualidade da LMX, indicando a relação positiva de percepção de dissimilaridade e a qualidade do relacionamento. Igualmente indicam que não há relação entre similaridade/dissimilaridade real e a qualidade dessa relação. Tais resultados são congruentes com a proposição de que as pessoas agem com base na percepção da realidade e não na realidade propriamente dita. Ênfase deve ser dada à similaridade percebida de acordo com o aspecto idade. Enquanto a idade é um dado absoluto, sua percepção é influenciada pelo compartilhamento de experiências da geração, data de entrada na organização e situações lá vivenciadas, representatividade numérica etc. (Markert, 2004; Pfeffer, 1985).

Como já mencionado, a percepção de similaridade resulta de inúmeros fatores que contribuem para destaque de determinadas características, em detrimento de outras. Assim, pode-se concluir que atributos reais como gênero, idade, ou até conflitos entre família e trabalho não são características para a percepção de similaridade entre superiores e subordinados e não exercem influência na qualidade da relação.

A hipótese do contato propõe que relações interpessoais entre representantes de grupos diferentes podem evoluir por conta da percepção de similaridade, o que leva a descategorização de determinados grupos e recategorização em outros em que ambos sejam identificados como pertencentes (Miller, 2002; Pettigrew, 1998). Sob a ótica da LMX, a relação entre superiores e subordinados pode ou não evoluir para uma relação diádica de qualidade, com base no reconhecimento de grupo de pertencimento. Ademais, se há percepção de similaridade, o contato tende a ser satisfatório, retroalimentando e ampliando a percepção de similaridade e assim em diante. A satisfação em relação ao contato pode ser observada em duas dimensões: qualidade e frequiência do contato (Triandis, 1995).

Verificou-se que tanto Qualidade como Freqüência do Contato são variáveis que alteram a relação observada entre as variáveis independentes e a variável dependente, melhorando a qualidade preditora das primeiras sobre a segunda. O efeito moderador observado na satisfação com a qualidade / freqüência do contato na relação é consistente, verificável tanto na matriz de correlação como nas análises de regressão hierárquicas, podendo-se questionar se estas são variáveis moderadoras ou se agem de fato como variáveis independentes. Entretanto, intrínsecas à Teoria da LMX, algumas condições também são pertinentes ao desenvolvimento de contato satisfatório, como o respeito, a confiança e as trocas e obrigações mútuas, sendo possível que ambas as variáveis estejam fortemente associadas, mas não necessariamente, em relação de dependência.

Os resultados deste estudo conduzem a um paradoxo. Enquanto na prática o ser humano é atraído por seu semelhante, e quanto mais semelhante o percebe, melhor se relaciona com ele, o discurso da literatura recente aponta os ganhos que se obtêm por meio da diversidade, quando bem administrada (Cox, 1991, 2002; Cox \& Blake, 1991; Thomas, 1991). Além disso, a diversificação das pessoas dentro das organizações é fato inexorável. Cada vez mais mulheres ingressam no mercado de trabalho e desenvolvem carreiras para posições de comando. Os avanços na medicina e as melhorias das condições de vida permitem às pessoas atingir idades mais avançadas. Pessoas de diferentes origens étnicas e raciais buscam a igualdade de acesso às oportunidades de trabalho e o respeito às preferências sexuais é cada vez mais demandado. Por isso lidar com a diversidade e dela extrair os resultados mais positivos é quase um imperativo estratégico das organizações.

Mas não se jogue a toalha ainda. Os resultados também apontam a influência da satisfação com o contato na relação diádica. É possível ampliar a qualidade da relação entre superiores e subordinados com o desenvolvimento de práticas e políticas orientadas para a valorização das diferenças e comportamentos cooperativos, que promovam condições para o desenvolvimento de contatos mais satisfatórios. Tais condições são, de acordo com a Hipótese do Contato, a complementaridade e cooperação diádica, a superposição de metas, o suporte institucional e a igualdade de status (Pettigrew, 1998). A superposição de metas, por exemplo, que hoje é prática comum nas organizações, muitas vezes alinhada ao sistema de recompensas, além de promover o alinhamento estratégico de metas, pode contribuir para a melhoria da relação, por meio da ampliação da percepção de similaridade, independentemente de similaridade e dissimilaridade real. 
Os resultados indicam que a diversidade das díades, nas dimensões de gênero e idade, não resultam em efeitos positivos ou negativos para a qualidade do relacionamento. Tais resultados vão ao encontro dos paradigmas subjacentes às práticas de gestão mais recorrentes do cenário nacional, voltadas para a promoção de justiça, ampliação da representação e redução de preconceitos e discriminação (Hanashiro \& Carvalho, 2005). Pela ótica da identidade cega (identity-blind), tais práticas enfatizam as realizações dos indivíduos, a despeito do grupo de identificação. Este paradigma da diversidade traz como característica a baixa incidência de conflitos entre grupos de identidades diferentes, na medida em que não há saliência para as diferentes identidades. Por outro lado, é do conflito de idéias, pela identificação das assimetrias entre si mesmo e os demais que ocorrem as mudanças das idéias que podem levar à evolução delas (Geertz, 2001).

\section{LIMITAÇÕES E NOVAS PESQUISAS}

Em decorrência da amostra de conveniência aliada à necessidade de complementação diádica dos questionários, pode ter havido prevalência de respondentes com melhor qualidade de relacionamento, visto que díades com baixa qualidade de relação podem não ter retornado seus questionários.

Não há indicações de que a Escala LMX-7 tenha sido usada anteriormente no Brasil. Os resultados deste estudo são suficientes para validá-la para esta amostra; contudo novas pesquisas com sua utilização serão necessárias para confirmá-la como instrumento eficiente de avaliação do relacionamento de superiores e subordinados como base para a liderança efetiva.

Dada a ausência de escala para medir percepção de similaridade, optou-se por desenvolver algumas questões. A abordagem de percepção de similaridade fez-se por meio da investigação de similaridade no jeito de pensar em relação a características específicas. Essa forma de abordar pode ter gerado redundância de itens.

Os respondentes são profissionais de organizações localizadas em alguns municípios dos Estados de São Paulo e Paraná, o que pode não representar uma amostra da cultura brasileira, mais coletivista e com maior distância de poder (Hofstede, 1984), cujos relacionamentos diádicos e igualdade de status podem ter menos importância, sugerindo a continuidade do estudo com pesquisas que levem em conta traços da cultura brasileira.

Artigo recebido em 28.08.2007. Aprovado em 24.06.2008.

\section{REFERENNCIAS BIBLIOGRÁFICAS}

Aron, A. S., Steele, J. L., Kashdan, T. B., \& Perez, M. (2006). When similars do not attract: tests of a prediction from the self-expansion model. Personal Relationships, 13(4), 387-396.

Aronson, E., Wilson, T. D., \& Akert, R. M. (2002). Psicologia social (3a ed.). Rio de Janeiro: LTC Livros Técnicos.

Ashfort, B. E., \& Mael, F. (1989). Social identity theory and the organization. The Academy of Management Review, 14(1), 20-39.

Bauer, T. N., \& Green, S. G. (1966). Development of leader-member exchange: a longitudinal test. Academy of Management Journal, 39(6), 1538-1567.

Brislin, R. W. (1970). Back-translation for cross-cultural research. Journal of Cross-Cultural Psychology, 1(3), 185-216. 
Byrne, D. (1971). The attraction paradigm. New York: Academic Press.

Cortez, J. V. (2000). The development of leader-member exchange relationships and the effect of race and gender on these relationships. Doctoral dissertation. California School of Professional Psychology, Los Angeles, CA. USA.

Cox, T. H. (1991). The multicultural organizational. Academy of Management Executive. 5(2), 34-17.

Cox, T. H., \& Blake, S. (1991). Managing cultural diversity: Implications for organizational competitiveness. Academy of Management Executive, 5(3), 45 - 56.

Cox, T., Jr. (2002). Taking diversity to the next level. Executive Excellence, 19(4), 19.

Davis, D. D., Selgrade, K., Downey, H., \& Bryant, J. (2004, April). Leader-member exchange, workfamily conflict, and performance among teleworkers. Annual Meeting of the Society for Industrial and Organizational Psychology, Chicago, IL

Deluga, R. J. (1998). Leader-member exchange quality and effectiveness rating: the role of subordinate-supervisor conscientiousness similarity. Group \& Organization Studies, 23(2), 189216.

Duarte, N. T., Goodson, J. R., \& Klich, N. R. (1994). Effects of dyadic quality and duration on performance appraisal. Academy of Management Journal, 37(3), 499-521.

Engle, E. M., \& Lord, R. G. (1997). Implicity theories, self-Schemas and leader-Member exchange. Academy of Management Journal, 40(4), 988-1010.

Ferdman, B. M. (1995). Cultural identity and diversity in organizations: bridging the gap between group differences and individual uniqueness. In M. M. Chemers, S. Oskamp, \& M. A. Constanzo (Eds.). Diversity in organizations: new perspectives for a changing workplace. Thousand Oaks, CA: Sage Publications.

Garcia, M. F. (2004). Antecedents of an interviewer's fit perceptions of an Applicant: the role of actual and perceived similarity. Doctoral dissertation. Texas A\&M University, College Station, Texas.

Geertz, C. (2001). Nova luz sobre a antropologia. Rio de Janeiro: Jorge Zahar.

Graen, G. B., \& Uhl-Bien, M. (1998). Relationship-based approach to leadership: development of leader-member exchange (LMX) theory of leadership over 25 years: applying a multi-level multi-domain perspective. In F. Dansereau \& F. J. Yammarino (Eds.). Leadership: the multiplelevel approach (Vol. 24, Part B). Stamford, CO: Contemporary and alternative.

Greene, C. N. (1972 ). Relationships among role accuracy, compliance, performance evaluation, and satisfaction within managerial dyads. Academy of Management Journal, 15(2), 205-215.

Greenhaus, J. H., \& Beutell, N. J. (1985). Sources of conflict between work and family roles. Academy of Management Review, 10(1). 76-88.

Hair, J., Jr, Anderson R., Tatham , R., \& Black , W. (2006). Análise multivariada de dados. Porto Alegre: Bookman.

Hanashiro, D. M. M., \& Carvalho, S. G. (2005). Diversidade cultural: panorama atual e reflexões para a realidade brasileira. Revista Eletrônica de Administração, 11(5), 1-21.

Harrison, D. A., Price, K. H., \& Bell, M. P. (1998). Beyond relational demography: time and the effects of surface and deep-level diversity on work group cohesion. The Academy of Management Journal, 41(1), 96-107. 
Similaridade e Dissimilaridade entre Superiores e Subordinados e suas Implicações para a

Haslam, S. A., \& Platow, M. J. (2001). Your wish is our command: the role of shared social identity in translating a leader's vision into follower's action. In M. A. Hogg \& D. J. Terry (Eds.). Social identity theory and organizational process. Philadelphia, PA: Psychology Press.

Hobman, E. V., Bordia, P., \& Gallois, C. (2004). Perceived dissimilarity and work group involvement: the moderating effects of group openness to diversity. Group \& Organization Management, 29(5), 560-587.

Hogg, M. A., \& Terry, D. J. (2000). Social identity and self-categorization processes in organizational contexts. The Academy of Management Review, 25(1), 121-140.

Hogg, M. A., \& Terry, D. J. (Eds.). (2001). Social identity theory and organizational process. Philadelphia, PA: Psychology Press.

Huang, X., \& Iun, J. (2006). The impact of subordinate-supervisor similarity in growth-need strength on work outcomes: the mediating role of perceived similarity. Journal of Organizational Behavior. 27(8), 1121-1148.

Instituto Brasileiro de Geografia e Estatística. (n.d). Indicadores IBGE - O trabalho a partir dos 50 anos de idade. Pesquisa mensal de emprego (Recife, Salvador, Belo Horizonte, Rio de Janeiro, São Paulo e Porto Alegre). Recuperado em 20 março, 2007, de http://www.ibge.gov.br/home/estatistica/indicadores/trabalhoerendimento/pme_nova/trabalho_5 0anos.pdf

Instituto Brasileiro de Geografia e Estatística. (2008). O trabalho da mulher principal responsável no domicílio. Pesquisa mensal de emprego. Recuperado em 20 março, 2007, de http://www.ibge.gov.br/home/estatistica/indicadores/trabalhoerendimento/pme_mulher/Supleme nto_Mulher_2008.pdf

Liden, R. C., \& Graen, G. (1980). Generalizability of vertical dyad linkage model of leadership. Academy of Management Journal, 23(3), 451-465.

Lynch, P. D. (2001). Benefits of the contact conditions for organizational diversity: Examining effects of intergroup dynamics, ethnic identity, and heterogeneity. Dissertation doctor. University of Delaware, Newark, DE, USA.

Machado, H. (2003). A identidade e o contexto organizacional: perspectiva de análise. Revista de Administração Contemporânea, 7(Edição especial), 51-73.

Markert, J. (2004). Demographics of age: generational and cohort confusion. Journal of Current Issues and Research in Advertising, 26(2), 11-25.

Medin, D. L., Goldstone, R. L., \& Gentner, D. (1990). Similarity involving attributes and relations: judgments of similarity and difference are not inverses. Psychological Science, 1(1), 64-69.

Miller, N. (2002). Personalization and the promise of contact theory. Journal of Social Issues, 58(2). 387-410.

Paschoal, T., \& Tamayo, A. (2005). Impacto dos valores laborais e da interferência família: trabalho no estresse ocupacional. Psicologia: Teoria e Pesquisa, 21(2), 173-180.

Pettigrew T. F. (1998). Intergroup contact theory. Annual Review Psychology, 49, 65-85.

Pettigrew T. F., \& Tropp, L. R. (2006). A meta-analytic test of intergroup contact theory. Journal of Personality and Social Psychology, 90(5), 751-783.

Pfeffer, J. (1985). Organizational demography: implications for management. California Management Review, 28(1), 67-81. 
Phillips, A. S., \& Bedeian, A. G. (1994). Leader-follower exchange quality: the role of personal and interpersonal attributes. The Academy of Management Journal, 37(4), 990-1001.

Pulakos, E. D., \& Wexley, K. N. (1983). The relationship among perceptual similarity, sex and performance ratings in manager-subordinate dyads. Academy of Management Journal, 26(1), 129-139.

Robbins, S. P. (2005). Comportamento organizacional. São Paulo: Prentice Hall Pearson.

Saltz, J. L. (2004). Beyond simple similarity: the relationship $f$ leader-follower personality fit with follower satisfaction with the leader and follower commitment to the organization. Doctoral Dissertation. University of Maryland, College Park, Annapolis, MD, USA.

Silva A. B. (2005). A vivência de conflitos entre a prática gerencial e as relações em família. Tese de doutorado não-publicada. Universidade Federal de Santa Catarina, Florianópolis, SC. Brasil.

Sloutsky, V. M. (2003). The role of similarity in the development of categorization. Trends in Cognitive Sciences, 7(6), 246-251.

Strauss, J. P. (1993). An examination of the relationship between actual and perceived personality similarity (between the rater and the rate), liking, and performance ratings. Doctoral dissertation. The University of Iowa, Iowa City, IA, USA.

Strauss, J. P., Barrick, M. R., \& Connerley, M. L. (2001) An investigation of personality similarity effects (relational and perceived) on peer and supervisor ratings and the role of familiarity and liking. Journal of Occupational and Organizational Psychology, 74(5), 637-657.

Thomas, R. R., Jr., (1991). Beyond race and gender: unleashing the power of your total work force by managing diversity. New York: AMACOM.

Thomas, D. A., \& Ely, R. J. (1996). Making differences matter: a new paradigm for managing diversity. Harvard Business Review, 74(5), 79-90.

Triandis, H. C. (1995). A theoretical framework for the study of diversity. In M. M. Chemers, S. Oskamp, \& S. M. A. Constanzo (Eds.). Diversity in organizations: new perspectives for a changing workplace. Thousand Oaks, CA: Sage Publications.

Triandis, H. C. (2003). The future of workforce diversity in international organizations: a commentary. Applied Psychology: An International Review, 52(3), 486-495.

Triandis, H. C. (2004). The many dimensions of culture. Academic commentary. Academy of Management Executive, 18(1), 88-93.

Turban, D. B., \& Jones, A. P. (1988). Supervisor-subordinate similarity: types, effects, and mechanisms. Journal of Applied Psychology, 73(2), 228-234.

Turner, J. C. (1987), The analysis of social influence. In J. C. Turner, M. A. Hogg, P. J. Oakes, S. D Reider, \& M. S. Wetherell (Eds.). Rediscovering the social group: a self-categorization theory (pp. 42-67). Oxford: Blackwell.

Tsui, A. S., Egan, T. D., O'Reilly, C. A. III (1992). Being different: relational demography and organizational attachment. Administrative Science Quarterly, 37(4). 549-579.

Tversky, A. (2004). Preference, belief, and similarity: selected writings. Cambridge, MA: The MIT Press. 
Wexley, K. N., Alexander, R. A., Greenawalt, P., \& Couch, M. A. (1980). Attitudinal congruence and similarity as related to interpersonal evaluations in manager-subordinate dyads. Academy of management Journal, 23(2), 320-330.

Zebrowitz, L. A. (1990). Social perception. Buckingham: Open university press.

Yu, D., \& Liang, J. (2004). A new model for examining the Leader-Member Exchange (LMX) theory. Human Resource Development International, 7(2), 251-264.

Yukl, G. (2001). Leadership in organizations (5th ed.). Upper Saddle River, NJ: Prentice-Hall. 